\title{
Cannabidiol and Non-Steroidal Anti-Inflammatory Drug Interactions: A Case of Drug-Induced Aseptic Meningitis
}

\author{
Mallory Emiga, b, Jafar Kafaie ${ }^{\mathrm{a}}$, Samantha Ong ${ }^{\mathrm{a}}$, Xujia $\mathrm{Li}^{\mathrm{a}}$
}

\begin{abstract}
Cannabidiol (CBD) and other marijuana derivatives are being more widely used in the medical community by people in an attempt to alleviate a variety of symptoms. While these products have shown promise in their analgesic properties, little is known about the potential pharmacological interactions of these and other drugs. We present a case of a 57-year-old Caucasian woman who presented with altered mental status, ataxia, left-sided numbness, and slurred speech. An extensive workup was completed and found to be largely unremarkable, though a thorough history revealed that her symptoms were likely caused by concurrent use of CBD and non-steroidal anti-inflammatory drugs (NSAIDs) resulting in drug-induced aseptic meningitis. The benign nature of CBD makes it a promising avenue for pain relief. Physicians and patients should be informed about the potential drugdrug interactions of CBD and other medications.
\end{abstract}

Keywords: Cannabidiol; NSAIDs; Drug-induced aseptic meningitis

\section{Introduction}

Cannabidiol (CBD) is a phytocannabinoid that is derived from Cannabis sativa and Cannabis indica that has become more frequently used for its medicinal qualities. The consumer market of CBD-infused products is continuously growing, although its therapeutic benefits are still under question by the US Food and Drug Administration (FDA) [1]. Due to its low tetrahydrocannabinol (THC) concentration, CBD oils and other derivatives provide medicinal benefits without the psychoactive effects of recreational marijuana, one of the most commonly used illicit drugs in the USA [2]. CBD is generally used for the side effects of chemotherapy, pain, anxiety, and other neurological and psychiatric illnesses, including but not lim-

Manuscript submitted May 8, 2020, accepted June 5, 2020

Published online June 20, 2020

aSaint Louis University Hospital, Saint Louis, MO, USA

${ }^{\mathrm{b}}$ Corresponding Author: Mallory Emig, Saint Louis University Hospital, Saint

Louis, MO 63110, USA. Email: mallory.emig@health.slu.edu

doi: https://doi.org/10.14740/jnr598 ited to post-traumatic stress disorder, seizures, and Tourette's syndrome [3]. Generally, in the medical community, CBD is considered a low-risk drug and has been used for decades for its neuroprotective effects. However, CBD's actions throughout the body are not fully understood and there are many side effects that should be considered before prescribing to patients.

CBD extracts usually contain no or very low amounts of THC, which is the component in marijuana that induces the euphoric psychoactive effect. However, CBD still has side effects including diarrhea, nausea, irritability, and weight/appetite changes that should not be ignored [4]. More importantly, because CBD interacts with common biological targets implicated in drug metabolism and excretion, it increases the likelihood of drug-drug interactions (DDIs) with commonly prescribed and over-the-counter medications such as non-steroidal anti-inflammatory drugs (NSAIDs), antimicrobials, and antiepileptics [5]. The mean half-life of CBD is $2-5$ days, so the drug can cause effects even if the patient stopped using before any symptoms of DDIs present [6]. As CBD is implicated as both a subject and a cause of DDIs, physicians and patients should be made aware of potential safety concerns with CBD use.

The adverse drug effects and DDIs of CBD are based on its pharmacologic targets and pharmacodynamic qualities related to metabolism, absorption, and elimination. Molecular targets of CBD include a wide variety of receptors and channels, and its metabolites can act on a multitude of cytochrome P450 (CYP) enzymes. In particular, CYP enzymes are responsible for the metabolism of NSAIDs, one of the most widely used drugs worldwide. In the USA alone, more than 30 billion doses of NSAIDs are consumed and more than 70 million prescriptions are written for NSAIDs [7]. These drugs (e.g. ibuprofen, diclofenac, ketoprofen, naproxen, flurbiprofen, meloxicam, piroxicam, and tenoxicam) are metabolized by two enzymes of the CYP superfamily, mainly the CYP2C8 and CYP2C9 [8]. CBD decreases the activity of both CYP2C8 and CYP2C9 in vitro $[9,10]$. Because the activity of $\mathrm{CBD}$ on the enzymes in vivo has not been established, clinical data is needed to fully understand the DDIs between CBD and NSAIDs. Physicians should strongly consider DDIs when the use of CBD is reported in concurrence with the use of commonly prescribed medications such as NSAIDs.

Although studies have shown the various adverse effects of CBD on different organ systems, clinical data demonstrating the negative consequences of CBD DDIs are minimal. One prior clinical study by Geffrey et al presented DDIs between CBD and 
clobazam, supporting the fact that concomitant use of CBD with other medications should be monitored carefully [11]. In fact, though $\mathrm{CBD}$ has shown promising results in treating a variety of symptoms, its effects on different processes throughout the body remain poorly understood and therefore should be treated/recommended with caution. The current case study offers further evidence that DDIs between CBD and common medications such as NSAIDs can lead to unforeseen and potentially severe nervous system pathology such as aseptic meningitis.

\section{Case Report}

Our patient is a 57-year-old woman with a medical history significant for occasional headaches, thyroid nodules, a Warthin's tumor of the left parotid gland resected in 2009, coronary artery disease, hypertension, thyroid nodules, rheumatoid arthritis and a 40-pack-year smoking history, who presented with altered mental status, ataxia, left sided numbness, and slurred speech.

She initially presented to an outside facility with the above symptoms for 9 days. She underwent routine testing including magnetic resonance imaging (MRI) of her brain without contrast which was unremarkable. She was discharged home after 2 days. Two days after being discharged, she returned to the facility with agitation, aggressive behavior, and staring spells along with ongoing altered mental status and ataxia. She was somnolent and complained of headaches. She had no fever and routine labs were again unremarkable. She underwent a lumbar puncture (LP) and extensive lab testing, the results of which were largely non-specific. Urinalysis and toxicology were negative. There were no metabolic or vitamin abnormalities. Thyroid studies were significant for a thyroid stimulating hormone (TSH) of 0.18. Inflammatory markers were not elevated. Autoimmune panels were within normal limits. Human immunodeficiency virus (HIV) and rapid plasma reagent (RPR) for syphilis were non-reactive. Cerebrospinal fluid (CSF) analysis revealed a white blood cell (WBC) count of 180, 96\% lymphocytes, red blood cell (RBC) count of 5, glucose 62, protein 280 , and cytology and flow cytometry were negative. Comprehensive cultures were sent and were all negative. MRI total spine was done and MRI brain with and without contrast was repeated, this time showing diffuse leptomeningeal enhancement. Routine electroencephalogram (EEG) was unremarkable. She was transferred to our hospital for further evaluation.

Upon arrival at our facility, she was awake and alert without agitation. A detailed history was taken, which noted worsening of her intermittent headaches starting in April 2019. At that time, she began to use CBD oil for her rheumatoid arthritis along with her prescribed NSAIDs. Around that time, her headaches became more frequent and more painful, leading her to increased NSAID use. Over the next few months, she experienced persistent headaches and developed gait difficulties. The memory issues and confusion were the newest symptoms she experienced, leading to her wandering the streets around the neighborhood lost and confused. Upon further questioning, she recalled having episodes of subjective fevers and night sweats as well as unintentional weight loss of 25 LBS. Review of systems was otherwise negative.

She reported a family history of thyroid disease as well as rheumatoid arthritis. She denied a recent history of travel, dietary changes, and knowledge of any exposure to toxins. Neurological exam was non-focal and was significant for decreased orientation (only oriented to self and place), fatigue, and mild weakness. Palpation of the thyroid revealed an enlarged goiter. She had diffuse bruising of her extremities. No rashes were noted.

Over the next $24 \mathrm{~h}$, the patient's headaches worsened and she again became aggressive and agitated. She was started on hydrocodone-acetaminophen for headaches. She underwent thyroid ultrasound which showed a large multinodular thyroid as well as a second LP. LP showed protein 182, WBC 238 w/93\% lymphocyte, glucose 60, and flow cytometry and cytology were pending but eventually were unremarkable. Infectious labs were all negative. Encephalopathy, encephalitis, and paraneoplastic panels were sent out to Mayo clinic. The patient continued to have episodes of agitation and aggression, particularly at night, eventually requiring scheduled quetiapine. In the mornings, she had a poor recollection of the events that occurred overnight.

Due to high concern for an underlying malignancy, she underwent a whole-body positron emission tomography-computed tomography (PET-CT) which was significant for "intense fluorodeoxyglucose (FDG) uptake in the right parotid gland with subsegmental and tonsillar lymph nodes demonstrating increased FDG avidity concerning for malignancy". Hematology was consulted for the significant PET findings. They recommended a biopsy of the parotid gland. Due to her negative infectious workup and lack of fever, the patient was not started on antibiotics. An ear, nose, and throat (ENT) specialist was consulted for fine needle aspiration (FNA) of the right parietal mass which showed recurrence of a Warthin's tumor.

After discussion with the patient and family about the unclear etiology of her presentation, she denied leptomeningeal biopsy and the decision was made to trial a dose of intravenous (IV) steroids. She was started on IV methylprednisolone 500 $\mathrm{mg}$ twice a day (BID) for 3 days. She was given intravenous immunoglobulin (IVIG) $2 \mathrm{~g} / \mathrm{kg}$ in 2 days after receiving solumedrol for 3 days. The patient reported significant improvement in her headaches and cognition. She was able to be discharged home under the care of her family.

Encephalitis, encephalopathy, and paraneoplastic panels resulted in no abnormalities. She was seen in a follow-up clinic 1 month after being discharged and reported that she felt completely back to her baseline which was confirmed by family members. She underwent a follow-up MRI which showed no leptomeningeal enhancement or signal abnormality and no other findings.

\section{Discussion}

The patient's presentation and complicated medical history led to a concern for a wide range of etiologies. Malignancy was high on the differential given her smoking history and B symptoms. Metabolic disorders were also considered though it was difficult to pursue this differential with a lack of abnormal lab findings. Careful history taking helped support the possibility of medication-induced encephalitis given that her headaches 
worsened with an increase in the use of her NSAIDs and subsequent administration of topical CBD. Infection was considered, but the patient was never febrile while in the hospital and she did not improve with antibiotics. Extensive infectious labs were sent with no significant results, helping us rule out this possibility. Because of all the negative lab findings, a diagnosis of drug-induced aseptic meningitis was highly considered.

The current case study found that the concomitant use of CBD with meloxicam could have precipitated drug-induced aseptic meningitis (DIAM). DIAM is a rarer cause of meningitis that is associated with NSAID use [12]. The symptoms of DIAM are similar to those that are found in cases of meningoencephalitis, including fever, headache, altered mental status, arthralgia, and myalgia [13]. The diagnosis of DIAM is a diagnosis of exclusion and is made by ruling out other possible causes of meningitis. The fact that all the laboratory tests, in this case, were negative for malignancy, infections, and autoimmunity and she returned to her baseline with complete resolution of the brain MRI findings, supporting the likelihood that our patient's aseptic meningitis was caused primarily by the use of medications. The pathogenesis of DIAM can be attributed to two proposed mechanisms, one being direct meningeal irritation by the drug and the other being a hypersensitivity reaction to the drug [14].

Although there are no published clinical cases about meloxicam specifically inducing aseptic meningitis, NSAIDs have been shown to be related to this pathology [12]. Therefore, it is possible that meloxicam can have similar effects as other NSAIDs that have been shown to cause DIAM. Meloxicam is primarily metabolized to a 5'-hydroxymethyl metabolite by CYP2C9 (major) and CYP3A4 (minor) [15]. As stated before, $\mathrm{CBD}$ decreases the activity of CYP2C9 in vitro. It is possible that the inhibitory effect of CBD on CYP2C9 led to a higher concentration of meloxicam, which led to the DIAM. Given the increasing popularity of CBD, it is important for both physicians and patients to be aware of possible DDIs of these substances and the symptoms that may result.

\section{Acknowledgments}

None to declare.

\section{Financial Disclosure}

None to declare.

\section{Conflict of Interest}

None to declare.

\section{Informed Consent}

Consent to write and publish the case was obtained from the patient.

\section{Author Contributions}

ME provided the body of the case report and summarized the case as well as contributing to the discussion, revising the final draft, and submitting the final paper for review. JK revised the final draft and provided expertise on the clinical importance of the case. SO and XL drafted the Introduction and Discussion and compiled and reformatted the references section.

\section{Data Availability}

The authors declare that data supporting the findings of this study are available within the article.

\section{References}

1. U.S. Food and Drug Administration [Internet]. cited on May 3, 2020. What you need to know (and what we're working to find out) about products containing cannabis or cannabis-derived compounds; [about 4 screens]. Available from: https:/www.fda.gov/consumers/consumerupdates/what-you-need-know-and-what-were-workingfind-out-about-products-conta.

2. Results from the 2015 national survey on drug use and health: detailed tables. 2015. Accessed on March 14, 2020. https://www.samhsa.gov/data/sites/default/files/ NSDUH-DetTabs-2015/NSDUH-DetTabs-2015/NSDUH-DetTabs-2015.pdf.

3. Tzadok M, Uliel-Siboni S, Linder I, Kramer U, Epstein O, Menascu S, Nissenkorn A, et al. CBD-enriched medical cannabis for intractable pediatric epilepsy: The current Israeli experience. Seizure. 2016;35:41-44.

4. Iffland K, Grotenhermen F. An update on safety and side effects of cannabidiol: a review of clinical data and relevant animal studies. Cannabis Cannabinoid Res. 2017;2(1):139-154.

5. Brown JD, Winterstein AG. Potential adverse drug events and drug-drug interactions with medical and consumer Cannabidiol (CBD) use. J Clin Med. 2019;8(7):989.

6. Consroe P, Kennedy K, Schram K. Assay of plasma cannabidiol by capillary gas chromatography/ion trap mass spectroscopy following high-dose repeated daily oral administration in humans. Pharmacol Biochem Behav. 1991;40(3):517-522.

7. Medscape [Internet]. 1994-2020. cited on May 3, 2020. Nonsteroidal Anti-inflammatory Drug (NSAID) Toxicity; [about 2 screens]. Available from: March 13, 2020. https://emedicine.medscape.com/article/816117-overview.

8. Jaja C, Bowman L, Wells L, Patel N, Xu H, Lyon M, Kutlar A. Preemptive genotyping of CYP2C8 and CY$\mathrm{P} 2 \mathrm{C}$ 9 allelic variants involved in NSAIDs metabolism for sickle cell disease pain management. Clin Transl Sci. 2015;8(4):272-280.

9. Yamaori S, Koeda K, Kushihara M, Hada Y, Yamamoto I, Watanabe K. Comparison in the in vitro inhibitory effects of major phytocannabinoids and polycyclic aromat- 
ic hydrocarbons contained in marijuana smoke on cytochrome P450 2C9 activity. Drug Metab Pharmacokinet. 2012;27(3):294-300.

10. Highlights of prescribing information. 2018. Accessed on March 29, 2020. https://www.epidiolex.com/sites/default/files/EPIDIOLEX_Full_Prescribing_Information. pdf.

11. Geffrey AL, Pollack SF, Bruno PL, Thiele EA. Drug-drug interaction between clobazam and cannabidiol in children with refractory epilepsy. Epilepsia. 2015;56(8):12461251.

12. O'Brien WM, Bagby GF. Rare adverse reactions to nonsteroidal antiinflammatory drugs. J Rheumatol. 1985;12(1):13-20.

13. Tattevin P, Revest M, Lavoue S. Meningites et meningoencephalitesaseptiques. Reanimation. 2008;17(7):639650 .

14. Jolles S, Sewell WA, Leighton C. Drug-induced aseptic meningitis: diagnosis and management. Drug Saf. 2000;22(3):215-226.

15. Schmid J, Busch U, Heinzel G, Bozler G, Kaschke S, Kummer M. Pharmacokinetics and metabolic pattern after intravenous infusion and oral administration to healthy subjects. Drug Metab Dispos. 1995;23(11):1206-1213. 